\title{
Aspects cliniques et pratiques de la sédation consciente chez les patients ayant des besoins spécifiques
}

\author{
Martine Hennequin \\ Faculté de Chirurgie dentaire - Clermont Ferrand \\ Martine.hennequin@u-clermont1.fr
}

L'administration de psychotropes ayant pour effet de baisser la vigilance permet de faciliter l'accès aux soins dentaires pour les patients initialement peu coopérants. Les procédures de sédation les plus décrites en odontologie sont l'inhalation de mélange de protoxyde d'azote et d'oxygène et l'administration de benzodiazépines. Les troubles cognitifs, les troubles psychiques, l'immaturité développementale et certains troubles systémiques constituent des facteurs pouvant limiter l'efficacité et/ou la tolérance des procédures de sédation consciente. La connaissance de ces facteurs de risque peut permettre d'adapter les procédures de sédation aux besoins des patients. Cette présentation vise à décrire les indications et les limites des procédures de sédation consciente permettant de faciliter l'accès aux soins dentaires pour les patients en situation de handicap.

La sédation consciente par inhalation d'un mélange de protoxyde d'azote constitue le premier palier de sédation pour les soins dentaires. La procédure est largement utilisée pour ses propriétés analgésiques et sédatives ainsi que pour sa grande tolérance. Une étude française multicentrique conduite sur un groupe de 349 patients présentant des troubles cognitifs soignés sous inhalation d'un mélange équimolaire de protoxyde d'azote et d'oxygène (Kalinox ${ }^{\odot}$, Air liquide santé International), rapporte un taux d'efficacité de 91,4\%, l'absence d'effets indésirables graves, et $10 \%$ d'effets indésirables mineurs. Cependant, la prise en charge des patients présentant des troubles cognitifs, implique d'adapter l'accompagnement psycho-comportementales nécessairement associé à l'administration et permet d'optimiser le succès et la tolérance de la procédure.

L'administration de midazolam, benzodiazépine à demi-vie courte et fortement hypnotique, constitue le deuxième palier de sédation pour améliorer le vécu des soins dentaires. Quelques études rapportent les indications chez les patients à besoins spécifiques en bonne santé. L'administration intraveineuse offre le meilleur rapport efficacité/tolérance, mais ne peut être mise en œuvre en première intention chez tous les patients et doit souvent être associée soit à une administration per os, intra-rectale ou intra-nasal, soit à l'inhalation d'un mélange de protoxyde d'azote et d'oxygène. Une étude française menée auprès de 142 patients à besoins spécifiques soignés sous sédation par administration de midazolam décrit l'excellente efficacité de la procédure qui permet la réalisation d'au moins un acte dentaire dans $90 \%$ des cas. Elle montre également qu'aucun effet indésirable grave n'est survenu, mais que la prévalence des effets indésirables mineurs varie en fonction du profil psychologique du patient, atteignant $16,6 \%$ pour les patients ayant des troubles cognitifs et $6,8 \%$ pour les patients ayant des troubles anxieux.

Dans le contexte de l'harmonisation européenne, le développement des compétences du chirurgien dentiste en sédation conscient est nécessaire. Cette présentation argumente l'intérêt de la rédaction de recommandations consensuelles. 